\title{
Um Estudo Comparativo de Formas de Segmentação de Mercado: uma Comparação entre VALS-2 e Segmentação por Variáveis Demográficas com Estudantes Universitários
}

\author{
Alípio Ramos Veiga-Neto
}

\begin{abstract}
RESUMO
A segmentação de consumidores vem sendo utilizada no planejamento estratégico de empresas e instituições com objetivo de agrupar os consumidores segundo determinadas características que possam conferir homogeneidade a cada grupo. Este trabalho investigou a relação entre a segmentação por classes econômicas e a segmentação psicográfica, que agrupa os consumidores segundo suas atitudes, estilos de vida, comportamento de compra e características demográficas. A pesquisa utilizou como sujeitos um grupo de conveniência formado por 367 alunos de uma IES particular por considerar que são futuros profissionais que utilizarão técnicas de segmentação. Foi utilizado um instrumento, adaptado da proposta denominada VALS-2, do Instituto SRI, juntamente com o sistema de classificação ABIPEME. Como resultado, observou-se que, nesse grupo, as atitudes e estilos de vida se distribuíram verticalmente entre todas as classes econômicas detectadas no grupo. Conclui-se que, para o grupo testado, existe pouca relação entre esses dois tipos segmentação: a segmentação por classes econômicas oferece menores benefícios para esse mercado, a segmentação psicográfica pode ser ferramenta fundamental para a estratégia de marketing e que são necessários instrumentos de classificação psicográfica mais adaptados às particularidades do consumidor brasileiro.
\end{abstract}

Palavras-chave: psicografia; segmentação; demografia; classes socioeconômicas; VALS-2.

\begin{abstract}
The consumers' segmentation has been used on strategic planning of companies and institutions with the objective of containing the consumers in accordance with certain characteristics that can give homogeneity to each group. This work investigated a relationship among the economic classes segmentation and the psychographics segmentation, that puts on the same group consumers in accordance with their attitudes, lifestyles, purchase behavior and demographic characteristics. The research used as subjects a convenience group formed by 367 students of a private institution of higher education, considering this people are future professional that will use segmentation techniques. Was used a questionnaire adapted from VALS-2 of the SRI Institute, together with the classification system ABIPEME. As result, was found that, in that group, the attitudes and lifestyles were distributed in a vertical line among all the economic classes detected in the group. As conclusion, for the tested group, little relationship exists among those two types of segmentation, the segmentation for economic classes offers few benefits for that market, the psychographic segmentation can be primordial tool for the marketing strategy and are necessary more adapted instruments of psychographic classification to the Brazilian consumer's particularities.
\end{abstract}

Key words: psychographics; segmentation; demography; socioeconomic classes; VALS-2. 


\section{INTRODUÇÃO}

É comum encontrar na literatura acadêmica de administração, marketing e comportamento do consumidor as mesmas descrições de comportamento de consumidores relacionadas com a classe social a que possivelmente pertençam os sujeitos, como, por exemplo, em Kotler (1997) ou em Richers e Lima (1991). Mesmo nas recentes publicações especializadas em comportamento do consumidor, encontramos informações padronizadas no que respeita à categorização por classes sociais, as quais, nesse assunto, percebe-se que têm recebido pouca atualização das recentes pesquisas e contribuições científicas especializadas.

Entre as publicações recentes a respeito de comportamento do consumidor que apresentam a categorização em termos tradicionais de classes sociais destacamse Karsaklian (2000), Schiffman e Kanuk (2000) e, não menos importante e contendo um rico conteúdo em estudos de psicologia do consumidor, Sheth, Mittal e Newman (2001). Note-se que de acordo com tal categorização, "ao pé da letra", um consumidor que comprar mais um rádio, ou mudar para uma casa com mais um banheiro, passará a fazer parte de novo grupo social com comportamento bastante distinto do anterior. Em Sheth, Mittal e Newman encontra-se quase um capítulo inteiro em que se definem padrões de comportamento de cada uma das classes sociais de consumidores estadunidenses, ainda que nessa mesma publicação sejam encontrados também recentes estudos sobre valores, atitudes e estilos de vida de consumidores.

Giglio (2002) aborda essa questão mais criticamente, quando salienta que aspectos econômicos devem ser utilizados apenas para planejamentos macroeconômicos, ficando as estratégias de produto e serviços mais bem atendidas, quando amparadas em informações de ordem comportamental. Porém Giglio, citando Karsaklian, está desatualizado quando dedica meio capítulo ao sistema de segmentação VALS (Values and Life-Styles), sem fazer qualquer referência ao VALS-2.

As poucas novas propostas em segmentação de consumidores demandam esforços no sentido de selecionar, adaptar e utilizar instrumentos mais condizentes com as profundas alterações ocorridas quer no referencial teórico, quer na própria sociedade, que modificou substancialmente, nas últimas décadas, a concepção de diferenciações socioeconômicas. Nesse sentido, uma contribuição de fundamental importância e que, no entanto, tem tido pouca ou nenhuma repercussão 
na literatura psicológica brasileira é a de Mitchell (1983), que desenvolveu uma classificação da população dos Estados Unidos largamente utilizada hoje em dia naquele país, chamada VALS (Values and Life-Styles), e que vem merecendo divulgação e uso em outros países.

A fim de fornecer uma contribuição atual e fundamental em pesquisa empírica para o problema da detecção dos valores e estilos de vida característicos de estudantes de Instituições de Ensino Superior, estabeleceram-se como principais objetivos deste estudo os seguintes.

a) Classificar os sujeitos de acordo com o sistema VALS-2 bem como de acordo com o sistema ANEP/ABIPEME.

b) Identificar em alunos de ensino superior seus valores e estilos de vida e detectar se existe relação desses fatores com a classificação do sistema ANEP/ ABIPEME.

c) Verificar se existe relação entre o sistema de segmentação por classe econômica e o sistema de segmentação VAL-2.

\section{Conceito de Segmentação de Mercado}

Para que seja possível um melhor entendimento do conceito e da importância da segmentação, antes é necessária uma pequena recordação do conceito básico de 'marketing'. Marketing, segundo autores como Richers (1978), Kotler (1997) ou Cobra (1990), é um sistema administrativo que, por meio de um planejamento estratégico, procura conhecer as necessidades dos mercados alvo e atendê-las por meio da oferta de produtos ou serviços adequados a essas necessidades, tendo como resultado a possibilidade de atingir os objetivos da organização. A segmentação, nesse caso, é a base de toda a estratégia de marketing, porquanto primeiro se segmenta o mercado em grupos homogêneos de consumidores, escolhem-se aqueles grupos que oferecem melhores condições para serem atendidos com o tipo de serviço ou produto em que a organização é, ou pretende ser, especializada, de modo a atender às necessidades e desejos desses grupos específicos de consumidores. Em seguida posiciona-se o produto ou serviço, de forma a expressar suas diferenças e benefícios, para então decidir-se estrategicamente a melhor maneira de comunicar àqueles consumidores que suas necessidades podem ser, provavelmente, mais bem atendidas por esta organização. 


\section{A Evolução da Tipologia Demográfica para a Psicográfica}

As pesquisas de mercado costumam colecionar rotineiramente perfis como idade, renda, educação e outros fatores mensuráveis que possam indicar uma preferência por marca, produto, tipo de mídia ou diversão (Wells, 1975). Porém as informações demográficas apresentam várias limitações, por não apresentarem grupos homogêneos, conduzindo a possíveis simplificações e estereótipos. Dessa forma, suas informações não podem ser consideradas bom guia para decisões de mensagens de marketing, atendimento das necessidades de consumidores ou para mostrar como são seus estilos de vida e valores (Langer, 1985). A razão para uma pessoa comprar determinado produto ou marca, ou ter preferência por determinada mídia, vai além de qual idade essa pessoa tem, quanto dinheiro possui ou o que a pessoa faz (Bainbridge, 1999). A chave da estratégia de marketing está em descobrir a diferença entre o que os consumidores fazem e por que fazem (Booth, 1999). Foi somente depois dos agitados anos sessenta que surgiram novas propostas de estratégias de 'marketing de massa' e consequientes desenvolvimentos de métodos de medir os valores e estilos de vida de consumidores (Heath, 1995).

Entre as propostas de segmentação mais focadas no comportamento de compra dos consumidores destaca-se a psicográfica que, de acordo com Demby (1994), é um método de classificar pessoas por meio de somatório de informações, que incluem, além dos dados pessoais como idade, sexo, renda e profissão, também outros relacionados a suas atitudes e estilo de vida, isso em substituição às meras informações demográficas. De acordo com Demby, o termo deve ser usado para combinar fatores psicológicos, sociológicos e antropológicos tais como autoconceito e estilo de vida, usados para segmentar mercados em relação a decisões de compra ou preferência por um ou outro tipo de mídia. Segundo Engel, Blackwell e Miniard (1995, p. 293) "as medidas psicográficas são mais abrangentes do que as demográficas, as comportamentais e as socioeconômicas" separadamente.

Heath (1995) ressalta que cada profissional de marketing define o que é a segmentação psicográfica de forma distinta. Eckman, Kotsiopulos e Bickle (1997) afirmam que a psicografia mede estilos de vida que são avaliados por atividades, interesses e opiniões, e que características psicográficas são mais efetivas do que as características demográficas. Silverberg, Backman e Backman (1996) atestam que tipologia psicográfica é certa maneira de descrever os consumidores e determinar novas tendências de consumo. Booth (1999) descreve a psicografia como o estudo do comportamento de consumidores, tentando averiguar as motivações que levam consumidores a tomarem suas decisões de compra. Wyner 
(1992) considera como psicográficas as classificações que tentam relacionar os vários tipos de personalidade por categorias de produtos. Wells (1975) salienta que segmentação psicográfica oferece algo além da segmentação demográfica, pelo fato de que sua amplitude permite alcançar um campo extenso de conteúdos, como atividades, interesses, opiniões, necessidade, valores, atitudes e características da personalidade.

\section{O Sistema VAlS-2 de Segmentação}

De modo coerente com as profundas transformações ocorridas mundialmente no âmbito da segmentação em termos de status socioeconômico ou de atividades profissionais e no que respeita à mobilidade social, fazia-se necessário, para fins de pesquisa de mercado, recorrer a critérios mais adequados do que os disponíveis até à época do surgimento do VALS. Ao invés de se concentrar apenas em um ou dois critérios (por exemplo, renda e profissão), o novo instrumento, apoiado em pesquisas realizadas, tendo como sujeitos milhares de consumidores estadunidenses, adotou uma concepção pluralista que se apóia principalmente em duas ordens de variáveis que originam seu título: valores que a pessoa mais preza (VA) e estilo de vida dessa mesma pessoa (life style, LS).

Desenvolvido por Arnold Mitchell (1983) no Stanford Research Institute (SRI), o primeiro sistema, denominado VALS, foi baseado em teorias de motivação e desenvolvimento psicológico, particularmente na teoria da hierarquia das necessidades de Maslow (1954). O sistema classificava os indivíduos em nove grupos com características próprias, organizados de forma ascendente, desde os 'sobreviventes', que representam em termos grosseiros os indivíduos da base da pirâmide social, até os 'integrados', que representam o ponto mais elevado da cadeia (Karsaklian, 2000; Mowen, 1993). Ainda dentro do mesmo sistema, e acompanhando a evolução ascendente das categorias, formam-se agrupamentos de categorias, tais como os dirigidos para as necessidades básicas (need driven), os direcionados pelo grupo social (other directed) e os direcionados por interesses próprios (inner directed), como se vê na Figura 1. 


\section{Figura 1: Os Nove Grupos da Classificação Original VALS}

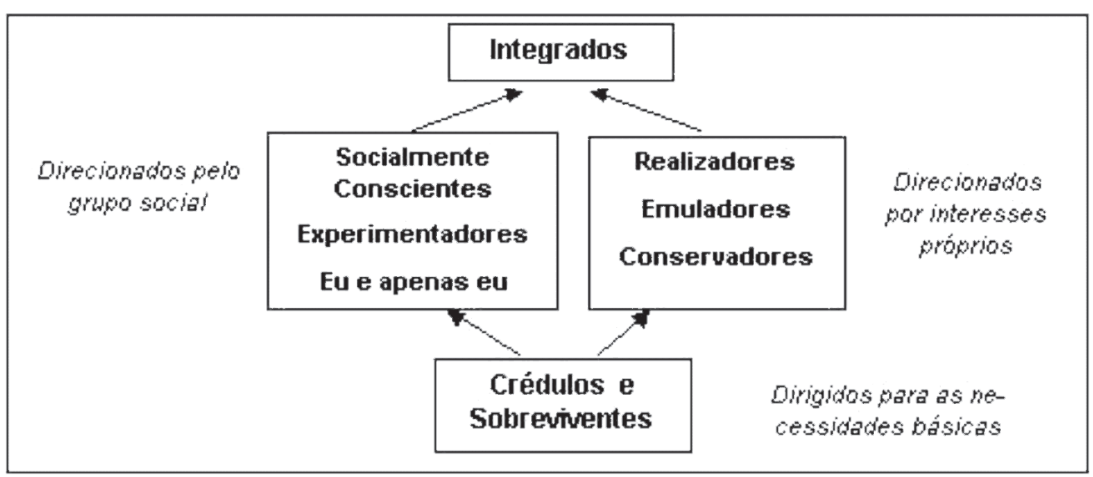

Fonte: (Karsaklian, 2000).

Durante a década de oitenta, várias empresas especializadas em pesquisa de mercado, usuárias regulares do sistema VALS, levantaram questionamentos sobre alguns elementos práticos no uso do sistema. O Stanford Research Institute recorreu a uma grande quantidade de informações acumulada nesses anos, que permitiram uma revisão e conseqüente evolução no sistema original de classificação VALS. Na versão revisada, o VALS-2, os pesquisadores do SRI enfatizaram menos os valores, demonstrando maior preocupação com as bases psicológicas do comportamento, influenciadas pelos recursos do consumidor.

Outras propostas de segmentação psicográfica foram apresentadas por vários pesquisadores. O Rokeach Value Survey identificou dois grupos de valores, terminais e instrumentais. Os valores terminais são os objetivos que buscamos na vida, idealizados para aferir a importância das expressões finais de existência, assim como uma vida próspera, um grande amor, respeito próprio. Os valores instrumentais são os padrões comportamentais pelos quais buscamos os valores finais de existência, assim como ter ambição, uma mente aberta, ser corajoso e generoso; portanto a primeira parte trata dos 'fins' e a segunda parte trata dos 'meios' (Rokeach, 1968, 1973). O LOV (List of Values) é instrumento de mensuração que também se destina a pesquisas de valores pessoais de consumidores. O sistema pede a consumidores que identifiquem em uma tabela de nove valores os dois com os quais mais se identificam. A lista é baseada nos valores terminais padronizados na Pesquisa Rokeach (Cannon, 1982; Kahle, Beatty, \& Homer, 1986; Kahle \& Kennedy, 1989; Kamakura \& Novak, 1992; Novak \& Macevoy, 1990; Pitts \& Woodside, 1983). O Yankelovich Monitor é um serviço comercial de pesquisa de mercado que sistematicamente rastreia tendências sociais. Sendo atualizado anualmente, foi inicialmente projetado para refletir vários valores culturais (Chisnall, 1995; Yankelovich, 1964, 2001). 
Assim como os anteriormente mencionados, vários outros sistemas foram propostos com maior ou menor repercussão, ou com alcance mais regionalizado tais como o Lifestyle Study da DDB (Piirto, 1990), o 4C's Cross Cultural Consumer Characterizations da Young \& Rubican (Piirto, 1990; Winters, 1992), e o AIO (Atividades, Interesses e Opiniões), que usa um questionário semelhante ao VALS, em que os pesquisadores apresentam uma série de frases sobre possíveis atividades, interesses e opiniões; as pessoas que respondem indicam se concordam com essas frases ou discordam (Wells \& Tigert, 1971).

\section{Grupos QUE COMPÕEM O VALS-2}

A versão em uso, na época desta pesquisa, do sistema do Instituto SRI, o VALS2, adota oito tipos para classificar os consumidores, conforme se vê na Figura 2. Desde o princípio de sua adoção, vem sendo utilizadas as seguintes denominações, no seu idioma original, para os tipos: Actualizer, Fullfilled, Achiever, Experiencer, Believer, Striver, Maker, Struggler. Essa proposta foi traduzida de inúmeras maneiras em diversas publicações editadas em português, causando muita confusão em relação a sua aplicação. Em Schiffman e Kanuk (2000) encontram-se as traduções Atualizado, Satisfeito, Empreendedor, Experimentador, Crente, Esforçado, Realizador e Batalhador. Em Sheth et al. (2001) foram traduzidos como Realizados, Satisfeitos, Realizadores, Experimentadores, Religiosos, Lutadores, Fazedores e Esforçados.

Karsaklian (2000) não faz menção ao VALS-2, sua descrição de tipos ainda é a do primeiro sistema VALS, dos anos oitenta. Giglio (2002) também se mostra desatualizado quando, citando Karsaklian e ignorando tanto outros autores como fontes disponíveis na Internet, dedica várias páginas ao antigo sistema VALS.

Em novembro de 2002, o Instituto SRI alterou sua página na Internet, modificando as denominações em inglês para três dos tipos VALS-2. O anteriormente denominado Actualizers passou a ser chamado Innovators. O tipo antes conhecido como Fullfilled passou a ser Thinkers. O tipo denominado Struggler foi mudado para Survivors. 
Figura 2: Diagrama Esquemático dos Oito Grupos do VALS-2

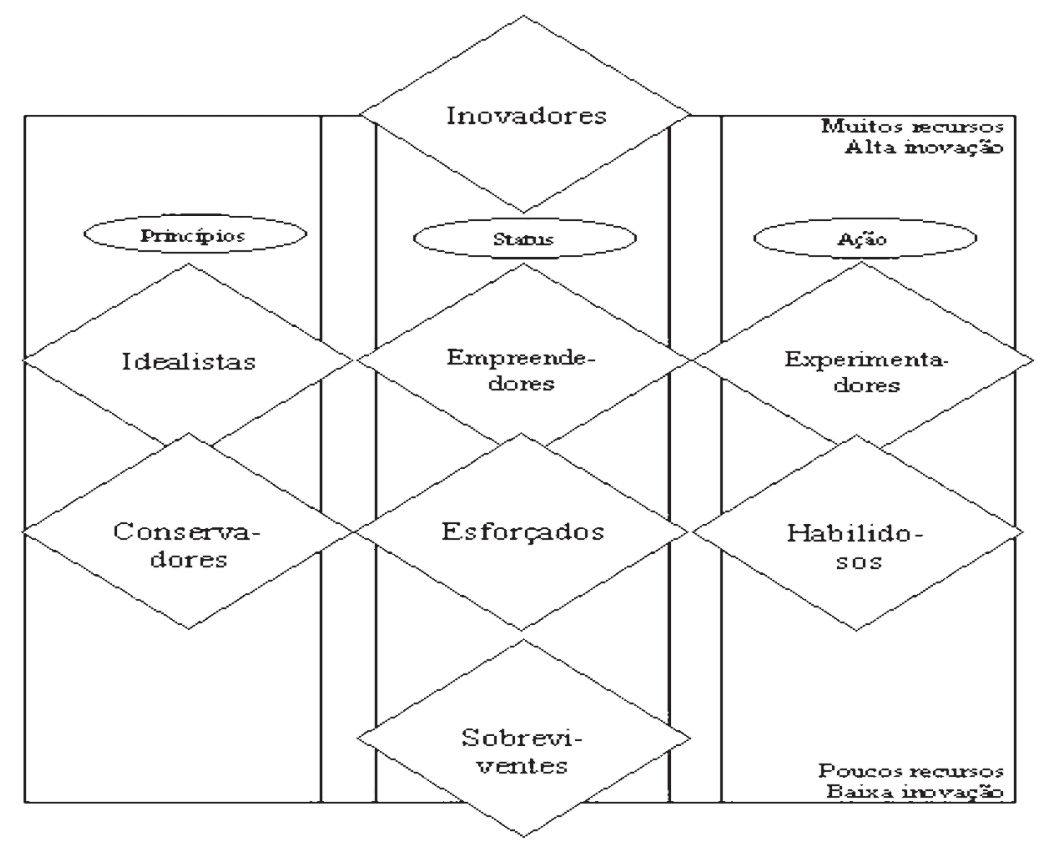

Fontes: Sheth et al. (2001); VALS Types (2002).

Considerando as dúvidas decorrentes das distintas traduções propostas nas literaturas disponíveis para a tipologia VALS-2, o Autor deste trabalho adotou uma proposta de tradução que, ainda que semelhante às suas equivalentes no idioma de origem, acredita que seja mais adequada à realidade da descrição dos tipos e não necessariamente à palavra empregada no idioma original. Dessa forma Innovators seria conhecido por Inovadores, Thinker poderia ser Idealista, Achiever tem uma versão adequada com Empreendedor e Experiencer equivale a Experimentador. A análise do termo Believer revela que aqui há uma problema mais sério, pois este não pode ser confundido com tipos apenas religiosos; dessa forma, as palavras religioso, crente ou crédulo não parecem expressar corretamente seu significado. Assim, sugere-se Conservador para definir esse tipo. Os últimos tipos são bem característicos. Dessa forma Striver pode ser Esforçado, Maker é bem característico de Habilidoso e Survivor é mesmo um Sobrevivente (VALS Types, 2002). A partir dessa proposta de tradução dos termos aqui mencionada, as denominações em português são adotadas no texto subseqüente deste artigo.

A seguir, é apresentada uma descrição aproximada das características dos indivíduos pertencentes a cada um dos grupos resultantes da segmentação VALS-2. Essa caracterização reflete os perfis dos cidadãos dos Estados 
Unidos da América (VALS Types, 2002). Não existem dados disponíveis que permitam traçar um paralelo desses perfis estadunidenses com indivíduos brasileiros.

Inovadores (Innovators): são bem sucedidos, sofisticados, ativos, pessoas com auto-estima elevada e recursos abundantes. Eles estão interessados em crescimento e buscam o autodesenvolvimento e o autoconhecimento, expressamse de vários modos, algumas vezes guiados por princípios, e outras vezes por um desejo de ação, desejo de fazer a diferença.

Imagem é importante para os Inovadores, não como evidência de status ou poder mas com o objetivo de saborear, de independência, de caráter. São lideres já estabelecidos ou emergentes em negócios ou no governo; contudo eles continuam buscando desafios. Têm um vasto campo de interesses, preocupamse com assuntos sociais e estão abertos a mudanças. Suas vidas são caracterizadas por riqueza e diversidade. Seus bens e tipo de recreação refletem gosto refinado para as melhores coisas da vida (Piirto, 1991).

Idealistas (Thinkers): são pessoas maduras, satisfeitas, apreciadores de conforto, refletem personalidades que estimam a ordem, o conhecimento e a responsabilidade. A maioria tem boa formação profissional (ou é aposentado recente). Eles estão bem informados sobre as notícias nacionais e mundiais. Estão despertos para oportunidades que permitam alargar seu conhecimento geral.

Felizes com sua carreira, família e estabilidade social, suas atividades de lazer tendem a concentrar-se nas proximidades de seu lar. Mostram respeito moderado pelo statu quo, autoridades e decoro social, mas são 'mente aberta' a idéias novas e mudanças sociais. Tendem a fundamentar suas decisões em princípios sólidos e por conseguinte aparentar tranqüilidade e segurança. Apesar de sua renda lhes permitir muitas escolhas, são consumidores conservadores, práticos; procuram durabilidade, funcionalidade e valor agregado nos produtos que compram.

Empreendedores (Achievers): gozam de uma carreira de êxito e são pessoas orientadas a escolher uma carreira de que gostem, o que geralmente fazem; querem ter controle sobre suas próprias vidas. Preferem o consenso e estabilidade e não gostam de correr riscos. Eles são profundamente dedicados ao trabalho e à família. $\mathrm{O}$ trabalho lhes proporciona senso de responsabilidade, recompensa financeira e prestígio. Suas vidas sociais refletem esse enfoque e são estruturadas ao redor da família, igreja e carreira. Vivem vidas convencionais, são politicamente conservadores e respeitam a autoridade e o statu quo. O que os outros pensam é muito importante, por isso adquirem produtos e serviços de prestígio que possam demonstrar seu sucesso. 
Experimentadores (Experiencers): são jovens, tem vitalidade, são entusiasmados, impulsivos e rebeldes. Buscam variedade e excitações, saboreiam o novo e apreciam o risco. Ainda no processo de formular seus estilos de vida e padrões de comportamento, ficam imediatamente entusiasmados com novas possibilidades, mas igualmente logo se desinteressam. Nesta fase de suas vidas, eles não são politizados, são ignorantes nesse sentido e altamente ambivalentes sobre aquilo em que acreditam.

Evitam estar em conformidade com a maioria, querem ser diferentes, mas invejam a riqueza, o prestígio e o poder de outras pessoas. São adeptos vigorosos da prática de exercícios físicos, esporte ao ar livre e atividades sociais. São consumidores ávidos e gastam muito de sua renda em roupas, fast-food, música, cinema e vídeo.

Conservadores (Believers): são pessoas conservadoras, convencionais, com convicções concretas, baseadas em códigos tradicionalmente estabelecidos, como família, igreja, comunidade e a nação. Muitos expressam códigos morais que estão profundamente arraigados e são literalmente interpretados. Eles seguem rotinas estabelecidas, realizadas em grande parte nas proximidades de seus lares, residem próximos de familiares e das organizações sociais ou religiosas a que pertencem. Como consumidores, são conservadores e previsíveis e favorecem produtos nacionais e marcas tradicionais. Sua renda e nível de educação são modestos, mas suficientes para satisfazer suas necessidades.

Esforçados (Strivers): estão em busca de motivação e da aprovação do mundo ao seu redor. Estão se esforçando para encontrar seu lugar na sociedade. São inseguros de si mesmos e possuem poucos recursos econômicos, sociais e psicológicos. Preocupam-se com as opiniões e a aprovação dos outros. Para eles, o sucesso se resume em possuir dinheiro, visto que não dispõem de reservas e freqüentemente sentem a vida de forma dura e amarga. São impulsivos e se ressentem facilmente. Muitos procuram vestir-se de acordo com a moda. São imitadores daqueles que consideram como ídolos, mas o que desejam obter está freqüentemente além de suas posses.

Habilidosos (Makers): são pessoas práticas que têm habilidades construtivas e conhecem seu valor pessoal. Vivem dentro de um contexto tradicional de família, emprego e recreação física. Têm poucos interesses fora desse contexto. Experimentam o mundo pelas mãos, construindo casa, criando crianças, consertando carro ou realizando pequenas atividades domésticas, como, por exemplo, fazer suas próprias conservas. Têm suficiente habilidade manual, renda e força de vontade para prosperar e concluir seus projetos. São politicamente conservadores, resistentes a novas idéias, respeitam a autoridade e o governo, mas ressentem-se quando estes invadem seus direitos individuais. Não se 
impressionam facilmente com produtos que não tenham um propósito prático ou funcional (como ferramentas, veículos utilitários e equipamentos de pesca).

Sobreviventes (Survivors): são pessoas de situação muito difícil, com uma vida de restrições. Têm pobreza crônica, baixo nível de educação e qualificação profissional, sem laços sociais fortes, displicentes com sua saúde e seu futuro, estão freqüentemente resignados e passivos. Pelo fato de estarem limitados pelas necessidades imediatas, mostram-se desorientados. Suas preocupações imediatas são a sobrevivência e segurança. São consumidores cautelosos. Representam um mercado muito modesto para a maioria dos produtos e serviços, mas são leais a suas marcas favoritas.

\section{Metodologia}

A fim de verificar que relações existem entre segmentação psicográfica e a segmentação por classes econômicas, optou-se por pesquisar um grupo de consumidores já segmentado demograficamente. Nesse grupo foi aplicado um questionário constituído por questões fechadas e abertas, para verificar a classe econômica a que pertence cada sujeito e a sua categorização VALS-2. A análise dos dados teve por base Siegel (1975); para fins de utilização de provas de hipóteses adequadas, foi empregado o Qui-quadrado, para análise intra e inter grupal. Tratase de uma pesquisa exploratória de natureza qualitativa, isto é, um tipo de pesquisa que tem como principal objetivo o fornecimento de critérios sobre a situação ou problema enfrentado pelo pesquisador e sua compreensão (Malhotra, 2001); porém foram conduzidas análises tanto quantitativas como qualitativas para cruzamento dos resultados. Foi utilizada a margem de significância de 0,05 , adequada para as ciências humanas.

\section{Participantes}

Este estudo concentrou-se na região de Campinas (Brasil). A pesquisa foi aplicada em uma Instituição de Ensino Superior da cidade de Americana, por pertencer esta última à macrorregião econômica de Campinas e ser uma cidade 'ilha' que recebe alunos de várias cidades circunvizinhas, formando assim uma amostra mais heterogênea de consumidores.

A amostra, por conveniência, foi constituída por 367 alunos da primeira à quarta série dessa instituição, pertencentes aos cursos de Comunicação Social e Turismo. Essa escolha se deveu à disponibilidade da amostra e ao fato de esses cursos corresponderem a atividades diretamente relacionadas com o atendimento ao 
consumidor em duas frentes distintas: na formação de opinião de consumo, pelos futuros profissionais de Comunicação Social, e no atendimento às suas exigências e particularidades de consumo, características dos futuros profissionais de Turismo. Os participantes foram subdivididos em dois grupos, formados pelos estudantes de Comunicação Social (GCS), composto por 240 participantes, e pelos estudantes de Turismo (GT), composto por 127 participantes.

\section{Material}

Foi utilizado um questionário, adaptado pelo Autor a partir de um sistema de investigação utilizado pela organização norte-americana de pesquisas SRI para pesquisa sobre valores, estilos de vida e bases psicológicas de consumidores. A versão original no idioma inglês desse questionário pode ser consultada em Sheth et al. (2001, p. 373) ou ainda na página na Internet da SRI (VALS Survey, 2002). Além desse instrumento, foram formuladas algumas questões com o objetivo de caracterizar os participantes, sendo também empregado um questionário simples, utilizado pela ANEP/ABIPEME, em que os participantes identificam o número de determinados itens que possuem em seus domicílios, para posterior cálculo da classificação econômica (ANEP, 2002).

O instrumento foi composto por questões abertas e fechadas, que permitiram verificar as concepções pessoais dos participantes; seu comportamento enquanto consumidores e usuários de produtos e serviços; e sua classe econômica.

\section{Procedimento}

A proposta foi apresentada aos Reitores de duas Instituições de Ensino Superior que se mostraram entusiasmados com a possibilidade de, além de poderem conhecer mais profundamente o perfil de seus alunos, contribuírem para o teste de um instrumento que atenda às novas exigências do INEP, quanto à adoção, pelas IES, de mecanismos de levantamento do perfil do aluno ingressante, que é avaliado pelas comissões de reconhecimento de cursos de graduação ou para fins de renovação de autorização. Uma das Instituições não pôde, infelizmente, ser incluída na pesquisa, apesar de contar com 18 mil alunos e situar-se em uma capital, porque está localizada fora do Estado de São Paulo. Limitou-se, pois, a pesquisa a uma única instituição, localizada em cidade prevista inicialmente. Após a obtenção da autorização do Reitor da Instituição e dos Coordenadores dos cursos a que pertencem os participantes, estes foram contatados pessoalmente pelo pesquisador e indagados a respeito de sua disposição no sentido de colaborar, sendo-lhes esclarecido que poderiam interromper a qualquer momento sua colaboração. A totalidade dos participantes contatados concordou em participar 
dos trabalhos de bom grado, mostrando-se até interessados em tomar conhecimento dos resultados da pesquisa.

Para averiguar possíveis falhas no instrumento ou mesmo dúvidas oriundas de seu preenchimento e para fins de orientação quanto à tabulação posterior, foi realizado uma pré-teste, com um grupo de 22 alunos.

\section{Resultados e Discussão}

Entre os especialistas de Marketing, nota-se que, mesmo depois de defenderem as claras vantagens de se conhecer o perfil psicográfico de consumidores, as empresas ou instituições que contratam serviços de pesquisa anseiam pelos resultados referentes às classes econômicas, na expectativa de que esses números vão auxiliá-las em tomadas de decisão. Parece apropriado, por conseguinte, iniciar a apresentação dos resultados da presente pesquisa pelos que se referem à categorização econômica dos sujeitos que, isoladamente, dão a impressão de serem números bastante confiáveis.

Cabe aqui um breve esclarecimento em relação ao número de participantes utilizados nos grupos correspondentes a cada curso e à sua totalidade. Como foi previamente observado, os grupos GT e GCS tiveram diferente número de sujeitos. A decisão no sentido de manter essa variação foi tomada após ponderação acerca de alguns aspectos importantes nesta investigação, entre os quais merecem realce: (a) a expectativa demonstrada pela reitoria da instituição em conhecer em números exatos o perfil de seus estudantes; (b) não fazer parte dos objetivos principais a comparação intergrupos, mas a verificação do sistema psicográfico de segmentação em comparação com o sistema de classes econômicas; e finalmente que (c), coincidentemente, o grupo GCS, com 240 participantes, é formado por praticamente o dobro dos sujeitos do grupo GT, com 127 participantes. Dessa forma, seria necessário eliminar $50 \%$ do total de participantes de um dos grupos por sorteio equiprovável, para obter-se um equilíbrio, que, neste caso, não pareceu ser desejável pelas razões já mencionadas.

Conforme pode ser verificado na Tabela 1 , os participantes parecem estar concentrados nas classes A2, B1 e B2. É fato que em investigações nas quais o sujeito deve identificar-se tendem a ocorrer inibições, quando este é questionado em relação à sua renda pessoal ou à renda de sua família. No caso da presente investigação, foi possível constatar total despreocupação dos participantes a este respeito, primeiro pela garantia de que não seriam identificados e depois pelo interesse que todos demonstraram em conhecer os resultados da investigação. 
Assim, pode-se supor que o nível de erro na declaração de renda seja mais ou menos equivalente ao nível de erro na declaração de bens que o sujeito possui, de modo que estaria confirmada a confiabilidade quanto ao sistema ANEP/ABIPEME de classificação econômica.

\section{Tabela 1: Distribuição por Classes Econômicas e Grupo a que Pertencem os Participantes}

\begin{tabular}{crrrrrr}
\hline Classe econômica & \multicolumn{2}{c}{ GT } & \multicolumn{2}{c}{ GCS } & \multicolumn{2}{c}{ Total } \\
& F & $\%$ & F & $\%$ & F & $\%$ \\
\hline A1 & 7 & 5,5 & 16 & 6,7 & 23 & 6,3 \\
A2 & 40 & 31,5 & 63 & 26,3 & 103 & 28,1 \\
B1 & 44 & 34,6 & 63 & 26,3 & 107 & 29,2 \\
B2 & 24 & 18,9 & 54 & 22,5 & 78 & 21,3 \\
C & 9 & 7,1 & 41 & 17,1 & 50 & 13,6 \\
D & 3 & 2,4 & 3 & 1,3 & 6 & 1,6 \\
E & 0 & 0,0 & 0 & 0,0 & 0 & 0,0 \\
Total & 127 & & 240 & & 367 & \\
X $^{2}$ & & 57,98 & & 33,32 & & \\
\end{tabular}

A fim de verificar em que extensão isto efetivamente ocorreu, a Tabela 2 apresenta a equivalência entre os níveis de renda declarada, utilizados no questionário VALS-2 e os níveis de renda que correspondem às diferentes classes econômicas. Outro ponto importante a ser observado é que os níveis de renda correspondentes às classes econômicas parecem ser os mesmos de 1997, por ocasião da proposta do Critério de Classificação Econômica Brasil, informados pela ANEP, com base em levantamento socioeconômico realizado pelo IBOPE em 1996 (ANEP, 2002). O Autor não encontrou na literatura especializada ou na Internet nenhuma atualização que levasse em consideração a inflação ocorrida nos últimos anos; haja vista que esse sistema leva em conta para o cálculo o poder de compra da renda doméstica do sujeito para adquirir os bens constantes do questionário, tais como TV, Videocassete, Rádio, Automóvel e empregada mensalista. 


\section{Tabela 2: Equivalência de Níveis de Renda Declarada pelos Sujeitos em Relação aos Considerados pela Classificação Econômica segundo o Critério ANEP - ABIPEME}

\begin{tabular}{|c|c|c|c|}
\hline Nível & Renda declarada & Classe equivalente & Renda respectiva \\
\hline 1 & Menos de $\mathrm{R} \$ 1.000,00$ & $\mathrm{Ce} \mathrm{D}$ & Abaixo de R\$ 1064,00 \\
\hline 2 & $\mathrm{R} \$ 1.000,00$ a $\mathrm{R} \$ 1.499,00$ & B2 & $\mathrm{R} \$ 1065,00$ a $\mathrm{R} \$ 1770,00$ \\
\hline 3 & $\mathrm{R} \$ 1.500,00$ a $\mathrm{R} \$ 1.999,00$ & B2 & $\mathrm{R} \$ 1065,00$ a $\mathrm{R} \$ 1770,00$ \\
\hline 4 & $\mathrm{R} \$ 2.000,00$ a $\mathrm{R} \$ 2.499,00$ & $\mathrm{~B} 1$ & $\mathrm{R} \$ 1771,00$ a $\mathrm{R} \$ 2943,00$ \\
\hline 5 & $\mathrm{R} \$ 2.500,00$ a $\mathrm{R} \$ 2.999,00$ & B1 & $\mathrm{R} \$ 1771,00$ a $\mathrm{R} \$ 2943,00$ \\
\hline 6 & $\mathrm{R} \$ 3.000,00$ a $\mathrm{R} \$ 3.999,00$ & $\mathrm{~A} 2$ & $\mathrm{R} \$ 2944,00$ a $\mathrm{R} \$ 5554,00$ \\
\hline 7 & $\mathrm{R} \$ 4.000,00$ a $\mathrm{R} \$ 4.999,00$ & $\mathrm{~A} 2$ & $\mathrm{R} \$ 2944,00$ a $\mathrm{R} \$ 5554,00$ \\
\hline 8 & $\mathrm{R} \$ 5.000,00$ a $\mathrm{R} \$ 7.499,00$ & A1 & Acima de $\mathrm{R} \$ 5555,00$ \\
\hline 9 & $\mathrm{R} \$ 7.500,00$ a $\mathrm{R} \$ 9.999,00$ & A1 & Acima de $\mathrm{R} \$ 5555,00$ \\
\hline 10 & $\mathrm{R} \$ 10.000,00$ a $\mathrm{R} \$ 19.999,00$ & A1 & Acima de $\mathrm{R} \$ 5555,00$ \\
\hline 11 & Acima de $\mathrm{R} \$ 20.000,00$ & $\mathrm{~A} 1$ & Acima de $\mathrm{R} \$ 5555,00$ \\
\hline
\end{tabular}

A partir da equivalência aqui proposta é possível ponderar acerca do nível de confiabilidade do sistema ANEP/ABIPEME em relação à renda declarada pelos participantes conforme a Tabela 3. De acordo com os dados contidos na Tabela 3 , do total de 367 participantes da presente pesquisa apenas 154 , ou seja, $42 \%$, declararam renda compatível com a classe econômica calculada a partir dos bens declarados, enquanto 213 participantes, ou 58\% do total, declararam renda inferior ou superior à classe econômica calculada pelo critério ANEP/ABIPEME.

\section{Tabela 3: Comparação da Classe Econômica Calculada pelo Critério ANEP/ABIPEME, com a Renda Declarada pelos Sujeitos}

\begin{tabular}{|c|c|c|c|c|c|c|c|c|c|c|c|c|c|c|}
\hline \multirow{3}{*}{$\begin{array}{l}\text { Nível de } \\
\text { renda }\end{array}$} & \multicolumn{12}{|c|}{ Classe econômica } & \multirow{2}{*}{\multicolumn{2}{|c|}{ E }} \\
\hline & \multicolumn{2}{|c|}{ A1 } & \multicolumn{2}{|c|}{ A2 } & \multicolumn{2}{|c|}{ B1 } & \multicolumn{2}{|c|}{ B2 } & \multicolumn{2}{|c|}{ C } & \multicolumn{2}{|c|}{ D } & & \\
\hline & $\mathrm{F}$ & $\%$ & $\mathrm{~F}$ & $\%$ & $\mathrm{~F}$ & $\%$ & $\mathrm{~F}$ & $\%$ & $\mathrm{~F}$ & $\%$ & $\mathrm{~F}$ & $\%$ & $\mathrm{~F}$ & $\%$ \\
\hline 1 & 0 & 0,0 & 0 & 0,0 & 3 & 2,8 & 4 & 5,1 & 15 & 30,0 & 6 & 100,0 & 0 & 0,0 \\
\hline 2 & 0 & 0,0 & 2 & 1,9 & 20 & 18,7 & 20 & 25,6 & 21 & 42,0 & 0 & 0,0 & 0 & 0,0 \\
\hline 3 & 0 & 0,0 & 5 & 4,9 & 12 & 11,2 & 18 & 23,1 & 6 & 12,0 & 0 & 0,0 & 0 & 0,0 \\
\hline 4 & 2 & 8,7 & 11 & 10,7 & 18 & 16,8 & 18 & 23,1 & 3 & 6,0 & 0 & 0,0 & 0 & 0,0 \\
\hline 5 & 2 & 8,7 & 11 & 10,7 & 19 & 17,8 & 9 & 11,5 & 2 & 4,0 & 0 & 0,0 & 0 & 0,0 \\
\hline 6 & 7 & 30,4 & 23 & 22,3 & 17 & 15,9 & 4 & 5,1 & 1 & 2,0 & 0 & 0,0 & 0 & 0,0 \\
\hline 7 & 0 & 0,0 & 23 & 22,3 & 9 & 8,4 & 2 & 2,6 & 2 & 4,0 & 0 & 0,0 & 0 & 0,0 \\
\hline 8 & 3 & 13,0 & 12 & 11,7 & 5 & 4,7 & 3 & 3,8 & 0 & 0,0 & 0 & 0,0 & 0 & 0,0 \\
\hline 9 & 4 & 17,4 & 10 & 9,7 & 3 & 2,8 & 0 & 0,0 & 0 & 0,0 & 0 & 0,0 & 0 & 0,0 \\
\hline 10 & 5 & 21,7 & 6 & 5,8 & 1 & 0,9 & 0 & 0,0 & 0 & 0,0 & 0 & 0,0 & 0 & 0,0 \\
\hline Total & 23 & 100,0 & 103 & 100,0 & 107 & 100,0 & 78 & 100,0 & 50 & 100,0 & 6 & 100,0 & 0 & 0,0 \\
\hline
\end{tabular}

(Observação: os números em negrito destacam os participantes cuja classificação em termos de classe econômica segundo ANEP/ABIPEBE coincide com a renda declarada).

Exposta a dúvida em relação à eficácia do sistema ANEP/ABIPEME, tratarse-á de considerar a segmentação psicográfica dos participantes baseada na tipologia VALS-2. Na tabulação realizada a este respeito foram adotadas as novas denominações propostas pelo Instituto SRI (VALS Types, 2002). Conforme pode 
ser verificado na Tabela 4, a tendência observada no pré-teste, de maior concentração de participantes no tipo Esforçado, confirmou-se em relação a 194 participantes, ou seja, 52,9\%, em relação a um total de 367 participantes. Observouse também números elevados de participantes nos tipos Empreendedor e Experimentador, com 61 e 86 participantes respectivamente, correspondentes aos percentuais de $15,6 \%$ e 23,4\%, enquanto os tipos Conservador e Habilidoso contaram com poucos representantes, 6 e 18 participantes respectivamente, ou seja, $1,6 \%$ e $4,9 \%$.

Acredita-se que esses números poderiam ser diferentes, se o grupo testado pertencesse a outros tipos de cursos, como, por exemplo, a área de engenharia, ou outro tipo de instituição de ensino superior, notadamente no caso das que pertencem à linha evangélica. De acordo com os perfis dos tipos VALS-2, não era esperado número significativo de sujeitos referente aos tipos Inovador e Idealista, fato que se confirmou. O número de participantes referente a estes tipos é quase nulo, ou seja, apenas um sujeito para cada tipo, representando $0,3 \%$ do total da amostra.

\section{Tabela 4: Distribuição dos Tipos VALS-2 por Curso a que Pertencem}

\begin{tabular}{lrrrrrr}
\hline \multicolumn{1}{c}{ Tipos VALS-2 } & GT & \multicolumn{2}{c}{ GCS } & \multicolumn{2}{c}{ Total } \\
& F & $\%$ & F & $\%$ & F & $\%$ \\
\hline Inovador & 0 & 0,0 & 1 & 0,4 & 1 & 0,3 \\
Idealista & 1 & 0,8 & 0 & 0,0 & 1 & 0,3 \\
Empreendedor & 19 & 15,0 & 42 & 17,5 & 61 & 16,6 \\
Experimentador & 23 & 18,1 & 63 & 26,3 & 86 & 23,4 \\
Conservador & 3 & 2,4 & 3 & 1,3 & 6 & 1,6 \\
Esforcado & 75 & 59,1 & 119 & 49,6 & 194 & 52,9 \\
Habilidoso & 6 & 4,7 & 12 & 5,0 & 18 & 4,9 \\
Sobrevivente & 0 & 0,0 & 0 & 0,0 & 0 & 0,0 \\
Total & 127 & & 240 & & 367 & 155,2 \\
$\mathrm{X}^{2} 0$ & & 144,0 & & 108,9 & & \\
\hline
\end{tabular}

Na comparação entre grupos GT e GCS, serão apresentados apenas os valores percentuais em relação ao número de participantes de cada curso, em virtude da diferença quanto ao número de participantes em cada curso. Volta-se a salientar que o principal objetivo desta investigação foi a verificação da aplicabilidade do VALS-2 em comparação com outros sistemas de segmentação. Dessa forma, ainda a Tabela 4 mostra que ambos os grupos, GT e GCS, mantiveram a tendência no sentido de maior concentração de participantes no tipo Esforçado (com 59,1\% para o GT e 49,6\% no GCS). Curiosamente, essa diferença para menos, no caso do GCS, parece ter sido acrescida aos tipos Empreendedor e Experimentador, resultando em $15 \%$ e $18,1 \%$ respectivamente, para o GT e 17,5 e $26,3 \%$, respectivamente, para o GCS. Os tipos Conservador e Habilidoso mantiveramse equilibrados, com $2,4 \%$ e $4,7 \%$ respectivamente para o GT e $1,3 \%$ e $5 \%$ 
respectivamente para o GCS. O tipo Inovador aparece com um sujeito no GCS, equivalente a $0,4 \%$, e o tipo Idealista aparece com um sujeito no GT, que equivale a $0,4 \%$.

Chega-se, assim, a um ponto essencial, na presente investigação: o da distribuição da segmentação psicográfica em relação à segmentação por classe econômica. Podem ser considerados aqui dois pontos de vista opostos. Um deles encara o perfil psicográfico de forma horizontal em relação às classes econômicas, ou seja, os indivíduos supostamente têm comportamento semelhante dentro de uma mesma classe econômica. Se isso é verdadeiro, então os segmentos psicográficos devem acompanhar essa tendência. O outro ponto de vista vê o perfil dos sujeitos de forma vertical em relação às classes econômicas, ou seja, os indivíduos são encarados mais como resultantes de seu envolvimento social (Allport, 1962), independentemente do nível econômico a que pertençam.

\section{Tabela 5: Distribuição dos Tipos VALS-2 por Classe Econômica na Totalidade dos Participantes}

\begin{tabular}{|c|c|c|c|c|c|c|c|c|c|c|c|c|c|c|c|c|}
\hline \multirow[b]{2}{*}{ Classe } & \multicolumn{2}{|c|}{ Inovador } & \multicolumn{2}{|c|}{ Idealista } & \multicolumn{4}{|c|}{$\begin{array}{l}\text { Classificação segundo o } \\
\text { Empreendedor Experimentador }\end{array}$} & \multicolumn{2}{|c|}{$\begin{array}{l}\text { VALS-2 } \\
\text { Conservador }\end{array}$} & \multicolumn{2}{|c|}{ Esforçado } & \multicolumn{2}{|c|}{ Habilidoso } & \multicolumn{2}{|c|}{ Sobrevivente } \\
\hline & $\mathrm{F}$ & $\%$ & F & $\%$ & $\mathrm{~F}$ & $\%$ & $\mathrm{~F}$ & $\%$ & $\mathrm{~F}$ & $\%$ & F & $\%$ & $\mathrm{~F}$ & $\%$ & F & $\%$ \\
\hline A1 & 0 & 0 & 0 & 0 & 7 & 11,5 & 8 & 9,3 & 0 & 0,0 & 7 & 3,6 & 1 & 5,6 & 0 & 0 \\
\hline $\mathrm{A} 2$ & 1 & 100 & 1 & 100 & 35 & 57,4 & 31 & 36,0 & 1 & 16,7 & 30 & 15,5 & 4 & 22,2 & 0 & 0 \\
\hline B1 & 0 & 0 & 0 & 0 & 14 & 23,0 & 19 & 22,1 & 1 & 16,7 & 65 & 33,5 & 8 & 44,4 & 0 & 0 \\
\hline B2 & 0 & 0 & 0 & 0 & 4 & 6,6 & 17 & 19,8 & 3 & 50,0 & 52 & 26,8 & 2 & 11,1 & 0 & 0 \\
\hline $\mathrm{C}$ & 0 & 0 & 0 & 0 & 1 & 1,6 & 11 & 12,8 & 1 & 16,7 & 34 & 17,5 & 3 & 16,7 & 0 & 0 \\
\hline $\mathrm{D}$ & 0 & 0 & 0 & 0 & 0 & 0,0 & 0 & 0,0 & 0 & 0,0 & 6 & 3,1 & 0 & 0,0 & 0 & 0 \\
\hline E & 0 & 0 & 0 & 0 & 0 & 0,0 & 0 & 0,0 & 0 & 0,0 & 0 & 0,0 & 0 & 0,0 & 0 & 0 \\
\hline Totais & 1 & & 1 & & 61 & & 86 & & 6 & & 194 & & 18 & & 0 & \\
\hline$X^{2} 0$ & & 0 & & 0 & & 99,2 & & 21,4 & & 33,33 & & 44,6 & & 45,1 & & 0 \\
\hline
\end{tabular}

A Tabela 5 permite a visualização dos resultados desta pesquisa em relação aos dois diferentes pontos de vista referidos no parágrafo anterior. Deve-se, inicialmente, desconsiderar o tipo Sobrevivente da proposta VALS-2 e a classe econômica $\mathrm{E}$, já que não ocorreu nenhum caso de participantes enquadrados nessas categorias. Conforme foi mencionado anteriormente, os tipos Inovador e Idealista do VALS-2 tiveram mínima representatividade e se localizaram na classe econômica A2, bastante adequado, levando-se em conta que a literatura sobre o VALS-2 posiciona esses tipos como pessoas que dispõem de fartos recursos. Porém, observados os outros tipos psicográficos, nota-se que os participantes se distribuem horizontalmente, independentemente da classe econômica, acompanhando assim a distribuição quantitativa anteriormente apresentada na Tabela 1, ou seja, há maior concentração de participantes na classes A2, B1 e $\mathrm{B} 2$, que se deve ao fato de haver proporcionalmente maior representatividade de sujeitos correspondentes a essas faixas. 


\section{Figura 3: Sobreposição da Distribuição dos Participantes nas Classes Econômicas e os Tipos VALS-2 mais Presentes}

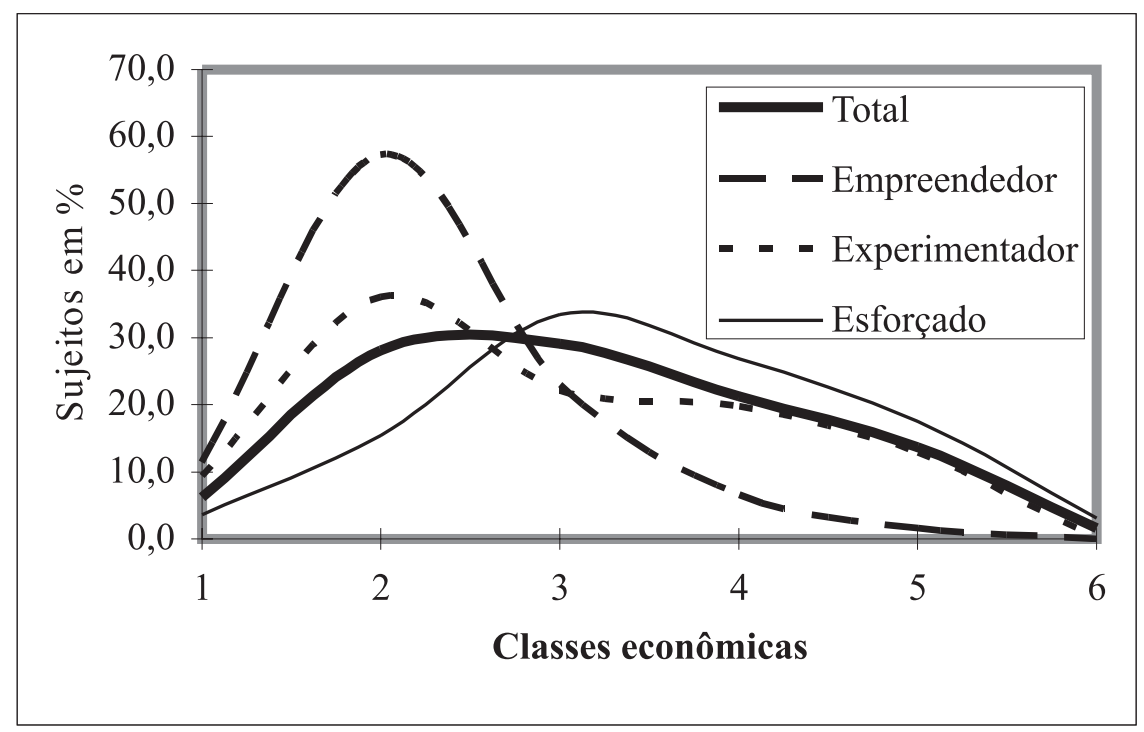

Para facilitar a visualização dessa situação, a Figura 3 apresenta um gráfico em que as curvas de percentuais de sujeitos enquadrados na tipologia VALS-2 acompanham a curva dos percentuais em relação ao total de sujeitos da pesquisa distribuídos entre as classes econômicas, permitindo melhor constatação da distribuição vertical dos participantes em relação à classe econômica.

\section{Conclusões}

O propósito principal desta pesquisa foi investigar a aplicabilidade de um sistema de segmentação baseado em informações relacionadas com as atitudes, estilo de vida e comportamento de consumidores e, além disso, comparar seus resultados com os de sistemas de segmentação meramente econômicos. Ao longo do processo de revisão da literatura, foi possível verificar que são poucas as informações científicas disponíveis sobre o tema no idioma português. A maioria do material reproduzido e ensinado nas Instituições de Ensino Superior a esse respeito reflete resultados de pesquisas realizadas com consumidores de outros países.

Com relação aos objetivos iniciais da investigação conclui-se que todos foram satisfatoriamente atingidos. Com relação ao primeiro objetivo, foi possível classificar todos os participantes tanto pelo sistema de classe econômica como 
pela segmentação psicográfica VALS-2. Porém, ainda que o instrumento VALS2 não seja totalmente adequado à realidade brasileira, surgiram maiores dúvidas no sistema ANEP/ABIPEME, pois muitos sujeitos não sabiam diferenciar modelos de geladeira e rádios ou se deviam considerar motocicleta e camioneta como carro. Com relação ao segundo objetivo, sua verificação foi possível por meio da análise dos grupos a que pertencem os sujeitos, ou seja, verificou-se tendências de compra, de acordo com os estilos de vida dos grupos a que pertenciam; porém, no grupo investigado, não foi possível verificar relação entre valores e estilos de vida com o sistema de classificação ANEP/ABIPEME. Finalmente, sobre uma relação entre o sistema VALS-2 e o sistema de classes econômicas, foi possível verificar que, nos sujeitos que participaram da presente pesquisa, parece haver pouca relação entre a classe econômica e os perfis psicográficos dos sujeitos, ou seja, o processo de decisão de compra ou as escolhas relacionadas com o consumo não dependem apenas do poder aquisitivo ou da classe econômica do consumidor, mas sofre fortes influências de grupos formadores de opinião e estão baseados, em grande parte, nas atitudes, estilo de vida e comportamento desse consumidor.

Esta investigação utilizou uma adaptação do sistema VALS-2, porém não teve, nem pretende ter, a intenção de defender esse como o único sistema a ser adotado no Brasil. A opção pela utilização desse sistema deveu-se ao fato de ser um sistema já testado por muitos anos em muitos milhares de pessoas, e à sua disponibilidade e facilidade de aplicação. Acredita-se que para ser adotado como padrão brasileiro esse sistema deveria sofrer adaptações nas tipologias que pudessem abranger mais adequadamente o perfil médio do consumidor do Brasil.

Muito embora seja necessário realizar mais investigações com o VALS-2 entre nós, junto a segmentos distintos da população, e aprofundar mais o nível da análise de sua natureza e implicações e aplicações, pode-se concluir que se trata de instrumento que, a exemplo do que ocorreu em outros países, contribui para uma avaliação e compreensão mais adequadas das características dos consumidores do que, por exemplo, a informação sobre nível de renda, que, isoladamente, oferece pouco para discernir bem as tomadas de decisão.

Têm ressonância indiscutivelmente positiva, no âmbito do marketing, os possíveis usos do VALS-2, em substituição (ou adicionalmente) a formas menos refinadas de classificação dos sujeitos, para fins de caracterização e diferenciação destes em pesquisas científicas, bem como para utilização por parte de profissionais de marketing e pesquisas de mercado, em processos de segmentação de consumidores, em complementação e cruzamento com outros dados mercadológicos demográficos e econômicos. 
Verificou-se, no levantamento bibliográfico, que, no mesmo tempo em que os institutos responsáveis pelo Critério de Classificação Econômica Brasil orientam enfaticamente seus associados para que adotem sua proposta de sistema, sem oferecerem argumentação de caráter científico comprobatório, existem pesquisadores, principalmente nas áreas de economia e administração (Mattar, 1996, 1997), que criticam esse sistema, comprovando, com base em pesquisas científicas, suas falhas. Apesar disso, o sistema é adotado até mesmo por instituições educacionais governamentais para, por exemplo, e supostamente, conhecer o estudante das IFES (Instituições Federais de Ensino Superior).

Por fim sugere-se para novas investigações que as pesquisas sejam focadas na criação ou adaptação de instrumentos de segmentação psicográfica mais adequados às particularidades do consumidor brasileiro, dentro de linhas de pesquisa que abracem o comportamento de consumidores, com o objetivo de formar docentes capacitados que possam aprofundar-se nessa área e contribuir tanto para o conhecimento do consumidor brasileiro como para a aplicação desse conhecimento na realidade brasileira.

\section{Artigo recebido em 26.08.2004. Aprovado em 18.08.2005.}

\section{RefERÊnCIAS Bibliográficas}

Allport, G. W. (1962).

Becoming: basic considerations for a psychology of personality. New Haven: Yale University Press.

Associação Brasileira das Empresas de Pesquisa - ANEP (2002).

Critério de classificação econômica Brasil. Recuperado em 16 maio, 2002, de http://www.anep.org.br

Bainbridge, H. (1999, June).

Beyond demographics. Wireless Review, 16(11), 61-62.

Booth, E. (1999, June).

Getting inside a shopper's mind. Marketing, 33-34. Recuperado em 25 novembro, 2001, de http:// www.accessmylibrary.com/coms2/ summary_0286-6647092_ITM

Cannon, H. M. (1982, September/ October).

A new method for estimating the effect of media context - using value profiles of ads. Journal of Advertising Research, 22(5), 41-48.

Chisnall, P. M. (1995).

Consumer behavior. New York: Prentice Hall.

Cobra, M. (1999). Administração de marketing (5a ed.). São Paulo: Atlas. 
Demby, E. H. (1994, Spring).

Psychographics revisited: the birth of a technique. Marketing Research, 6(2), 26-29.

Eckman, M.,

Kotsiopulos, A., \&

Bickle, M. C. (1997, February).

Store patronage behavior of hispanic versus non-hispanic consumers: comparative analyses of demographics, psychographics, store attributes, and information resources. Journal of Behavioral Sciences, 19(1), 69-84.

Engel, J. F.,

Blackwell, R. D., \&

Miniard, P. W. (1995).

Comportamento do consumidor. Rio de Janeiro: LTC.

Giglio, E. (2002).

O comportamento do consumidor. São Paulo: Pioneira.

Heath, R. P. (1995, November/December). Psychographics: Q'est-ce q c'est. Marketing Tools, 74(7), 74-81.

Kahle, L. R.,

Beatty, S. E., \&

Homer, P. (1986, December).

Alternative measurement approaches to consumer values: the list of values (LOV) and values and life style (VALS). Journal of Consumer Research, 13(3), 405-409.

Kahle, L. R., \&

Kennedy, P. (1989, December).

Using the list of values (LOV) to understand consumers. Journal of Consumer Marketing, 6(3), 5-12.
Kamahura, W. A., \&

Novak, T. P. (1992, June).

Value-system segmentation: exploring the meaning of LOV. Journal of Consumer Research, 19(1), 119-132.

Karsaklian, E. (2000).

Comportamento do consumidor. São Paulo: Atlas.

Kotler, P. (1997).

Administração de marketing (5a ed.). São Paulo: Atlas.

Langer, J. (1985, March).

Using psychographics to understand demographic groups. Marketing Review, 40(4), 11.

Malhotra, N. K. (2001).

Pesquisa de marketing - uma orientação aplicada (3a ed.). Porto Alegre: Bookman.

Maslow, A. H. (1954).

Motivation and personality. New York: Harper \& Row.

Mattar, F. N. (1996).

Porque os estudos de classificação socioeconômicos não funcionam no Brasil. Anais do Encontro Nacional dos Programas de Pós-Graduação em Administração, Angra dos Reis, RJ, 20.

Mattar, F. N. (1997).

Novo modelo de estratificação socioeconômica para marketing e pesquisas de marketing. Anais do Seminário em Administração FEAUSP, São Paulo, SP, 2. 
Mitchell, A. (1983).

The nine american life styles. New York: Macmillan.

Mowen, J. C. (1993). Consumer behavior. New York: Macmillan.

Novak, T. P., \&

Macevoy, B. (1990, June).

On comparing alternative segmentation schemes: the List of values (LOV) and values and life styles (VALS). Journal of Consumer Research, 17(1), 105-109.

Piirto, R. (1990).

Beyond mind games - The marketing power of psychographics. Ithaca, NY: Amer Demographics Books.

Piirto, R. (1991, July).

VALS the second time. American demographics, 13(7), 6-7.

Pitts, R. E., \&

Woodside, A. G. (1983, April).

Personal value influences on consumer product class and brand preferences. The Journal of Social Psychology, 119(2), 37-53.

Richers, R. (1978).

Ensaios de administração mercadológica. Rio de Janeiro: FGV.

Richers, R., \&

Lima, C.P. (1991).

Segmentação. Opções estratégicas para o mercado brasileiro. São Paulo: Nobel.

Rokeach, M. (1968).

Beliefs, attitudes, and values. São Francisco: Jossey Bass.
Rokeach, M. (1973).

The nature of human values. New York: The Free Press.

Schiffman, L. G., \&

Kanuk, L. L. (2000).

Comportamento do consumidor. Rio de Janeiro: LTC.

Sheth, J. N.,

Mittal, B., \&

Newman, B. I. (2001).

Consumer behavior and beyong. Orlando: Dryden Press.

Siegel, S. (1975).

Estatística não paramétrica para ciências do comportamento. São Paulo: McGraw Hill.

Silverberg, K. E.,

Backman, S. J., \&

Backman, K. F. (1996, Fall).

A preliminary investigation into the psychographics of nature-based travelers to the southeastern United States. Journal of Travel Research, 35(2), 19-28.

The VALS Segments - VALS Types (2002). SRI Consulting Business Intelligence. Recuperado em 10 outubro, 2002, de http://www.sricbi.com/VALS/types.shtml

The VALS Survey - VALS Survey (2002). SRI Consulting Business Intelligence. Recuperado em 15 novembro, 2002, de http://www.sricbi.com/VALS/presurvey.shtml

Wells, W. D. (1975, May).

Psychographics: a critical review. Journal of Marketing Research, 12(2), 196-213. 
Wells, W. D., \&

Tigert, D. J. (1971, August).

Activities, interests and opinions. Journal of Advertising Research, 11, pp. 27-35.

Winters, L. C. (1992, September).

International psychographics. Marketing Research, 4(3), 48-49.

Wyner, G. A. (1992, December).

Segmentation design. Marketing Research, 4(4), 38-41.
Yankelovich, D. (1964, March).

New criteria for market segmentation. Harvard Business Review, 42(2), 8390.

Yankelovich, D. (2001, March).

Yankelovich Monitor Mindbase. Recuperado em 25 novembro, 2001, de http://secure.yankelovich.com/ solutions/mindbase.asp 
